\title{
Designing for Pandemics - a Design Concept based on Technology Mediated Nudging for Health Behavior Change
}

\author{
My Zetterholm \\ Linnaeus University \\ my.zetterholm@1nu.se
}

\author{
Patrik Elm \\ Linnaeus University \\ patrik.elm@lnu.se
}

\author{
Sadaf Salavati \\ Linnaeus University \\ sadaf.salavati@lnu.se
}

\begin{abstract}
This paper addresses the Covid-19 pandemic and the need to find innovative approaches to fight transmission when societies open up. Throughout the pandemic, a number of countries have released mobile applications for contact tracing which has sparked a debate about privacy and ethics. To complement existing solutions, this paper proposes a different approach. This paper presents a design concept for an application promoting health behavior change based on Bluetooth proximity estimation and nudging theory. The approach is underpinned by current understanding of the main transmission routes, the risk of asymptomatic spreaders, and evidence of physical distancing to reduce transmission risk. The aim of this mobile system is to promote physical distancing, in line with public health guidelines promoted all over the globe. The concept stems from design thinking and a shift in perspective: from solutions focused on tracking infections to solutions focused on primary prevention by supporting human behavior.
\end{abstract}

\section{Introduction}

In December 2019 a cluster of pneumonia cases were discovered in Wuhan, China. These cases were caused by a newly identified coronavirus, later named as SARS-CoV-2 [1]. The virus rapidly spread over the world, and at the end of January 2020 the World Health Organization (WHO) officially declared the epidemic to be a public health emergency of international concern (PHEIC). By March 11th, the epidemic was updated to a pandemic, due to its spread across multiple continents [2]. This is the third time a new and highly pathogenic coronavirus causes an epidemic in the human population in the twenty-first century; the first being SARS-CoV in 2002, and the second, MERS-CoV in 2012 [1]. Furthermore, this is the sixth time a PHEIC has been declared in the past fifteen years [3], indicating that the current epidemic will not be the last. At the time of writing this paper (July 1st, 2020), the Coronavirus outbreak situation has reached 10,321,689 confirmed cases and 507,435 confirmed deaths globally. The virus has affected in total 216 countries, areas or territories according to WHO official statistics. Governments around the world responded to the pandemic in various ways: some countries implemented enforced lock down, with the intention of re-opening societies slowly. Others kept parts of their communities open but forbid crowding [4]. In May 2020, many nations started to re-open after a long quarantine, which has led to new outbreaks occurring in various countries [5]. As vaccines are not yet available, non-pharmacological approaches have been the only tools to tackle the spread. Now there is a need for additional innovations [6]. Some countries have turned to technology, such as smartphone-based applications, to facilitate contact tracing [7, 8, 9]. The main target of these mobile technological approaches have been disease orientated, and they have focused mainly on efforts to monitor symptoms or trace the spread of the pathogen. What is more, this development has sparked a debate about ethics and privacy [7, 8, 9]. This is the first pandemic taking place in a technological infrastructure where smartphone adoption is seen almost all over the world. This situation offers vast opportunities for ideation about how mobile technology can support public health strategies during epidemics in general, and in particular, in the current pandemic. As the area of using smartphones in epidemiology is still in its infancy, there is room for development [10]. The aim of this work is to contribute to design efforts striving to tackle the current pandemic by providing a new direction in which to understand and approach the problem; to inspire ideation about more human centered opportunities and to promote the development of technological solutions in respect of human rights. This approach is exemplified by a design concept. With the use of design thinking, we set out to alter the perspective and find a framework to enable more innovations to be made in this field. 


\subsection{Design thinking mindset}

As the area we are addressing requires an innovative approach our starting point has been to take a design thinking perspective into the problem domain. In this paper design thinking [11] is used at an abstract level as we apply a design thinking mindset [12] as framework for inspiration and a way of thinking. In this context it is represented by focusing on empathy as a driver for innovation, by approaching complex problems with no clear solution, by thinking outside the box and questioning the existing methods, by combining a number of scientific fields and thus utilizing creative thinking. This work includes elements of empathy, defining the problem (section two), and ideation (section three), which have been used to guide the focus of this work and the use of literature. Empathizing is at the core of this perspective, rather than a phase of its own. It drives the understanding of the problem, after both experiencing and listening to the human suffering caused by the pandemic expressed all over the globe. Empathy also inspired the purpose of this work, which strives to find more human-centered advancement with a less privacy invasive approach.

This work started with the question: how can we harness the inherent capabilities of mobile technologies in the context of infectious disease prevention? This starting point represents an expansion in thinking concerning how mobile solutions can produce value in pandemic contexts in order to look beyond the existing focus of how to design mobile contact tracing solutions. The latter is a digitalized version of a traditional manual method, which involves both practical and ethical challenges. Subsequently, the problem in this work was re-defined from a focus on tracing a pathogen towards a focus on how to model human spatial relations. Physical distance is promoted as one of the most important prevention measures during the current pandemic [13], but we have so far not seen enough (if any) scalable solutions to support people in this effort. By approaching the problem with a fresh perspective, we present a design concept in which the core idea is to use existing technology in new ways, which balances the ethical and practical constraints, and thus ensure the scalability needed in a pandemic context. In contrast to some of the existing mobile based solutions, the design concept proposed in this paper focuses on supporting human activities rather than tracking them, and thus the proposed system can act as a primary prevention tool. This can be one approach among many to support behavioral change and increase attention to distance as individuals all over the world needs to go back to their everyday lives.
The rest of the paper is structured as follows: section two focuses on the problem and provides a description of the pandemic situation. In section three a design direction is outlined, starting first by providing a theoretical background focused on behavioral change by nudging, and followed by a description of the design concept. The paper ends with plans for future research.

\section{Understanding a pandemic situation}

Using Reynold and Holwells [14] description of messy situations, epidemics and pandemics can be defined as messy and complex. Messy and complex situations involve many variables and they deal with multiple perspectives and uncertainties with no single correct solution or path to move forward. Understanding and defining the problem is complex due to the many elements, levels of interactions and relations. The rest of the section provides a description of the problem focusing on elements and their interactions in the current pandemic situation.

\subsection{The infectious agent, the host and the environment}

The main elements involved in epidemic situations are the infectious agent (the pathogen causing disease), the transmission process (how it spreads), the host (human or animal), and the environment (temperature, sanitation, access to health care or testing, and socio-economic aspects) [15]. Changes in any of these elements, or in the interactions between them, can affect how a virus spreads. For example, human activities such as social gatherings, agriculture or handling of animals, and natural events such as a genetic mutation in a virus, may cause a virus to change host or spread at a higher pace. Environments can affect the transmission in many ways and on many levels. Alteration in climate and the treatment of animals and nature may increase the risk of zoonotic viruses in a global context $[15,16]$. Socio-economic aspects, access to health care, testing capacity, infrastructure and access to information are all examples of contextual factors in the current pandemic. Furthermore, access to modern technological infrastructure may also offer opportunities not available in previous pandemics.

The infectious agent in the current pandemic is the virus SARS CoV-2, which causes various disease symptoms referred to as Covid-19 infection. SARS $\mathrm{CoV}-2$ is a zoonotic virus, meaning that it has transferred from animals to humans [17] - who are currently the main hosts and have spread the virus across the world [1].

The severity of Covid-19 disease varies to a great 
extent between individuals - from silent infections or very mild cold-like symptoms, to severe pneumonia or even acute respiratory distress. For some individuals, the disease is deadly [18]. The great variation in symptoms, and the fact that the disease causes mild infections in the majority of humans, enables many of the infected individuals to stay active and thus interact with others. There are also reports of transmission from individuals with no symptoms, which is discussed as a part of the explanation to why this corona virus spreads so easily [19]. Although there is still much to learn about transmission routes, the most considerable route is believed to be by droplets. These are drops from the nose and/or mouth of infected individuals, which land on surfaces or spread directly between individuals during close person-to-person interaction [18, 1, 13]. Transmission through aerosols is still discussed, but emerging evidence supports that this route exists [20, 21]. However, the majority of transmission takes place when there is a close and prolonged contact between individuals. Therefore, maintaining a physical distance is an important measure to reduce the risk of infection $[13,20]$. A recent review of the existing evidence on transmission routes shows that a physical distance between individuals of 1 meter can reduce the risk of transmission by $82 \%$. Moreover, increasing the distance up to 3 meters reduced the risk even further [21].

In situations such as the current Covid-19 pandemic, countries have applied multiple strategies to minimize transmission and flatten the epidemic curve. Social distancing has been key in the absence of pharmacological alternatives, and this will be described further in the upcoming subsection.

\subsection{Strategies and measures to reduce transmission}

Different strategies and measures have different consequences at various levels - for the individual, the population and the society as a whole. In the case of the current SARS CoV-2 virus, there is currently no vaccine available. Consequently, the tools that are available to tackle the situation have been centered around non-pharmacological approaches, and a key area of prevention is to minimize close contacts. This requires different methods depending on the political and cultural context. The variation in response strategies exemplifies the diversity in norms regarding the influence of governments and how to best regulate social behavior [4].

Quarantines, isolation and increasing the space between individuals is sometimes categorized under the umbrella term 'social distancing' [22, 23]. Quarantine refers to isolating individuals who may have been exposed. Isolation refers to separating individuals when disease has been confirmed [23]. Testing and contact tracing can assist this work by finding and isolating individuals who may have been exposed or have a confirmed infection. If exposed individuals are not easily identified, or contact patterns are not known, a community containment or nationwide quarantine may be needed. This can be implemented at various levels by closing certain environments and venues or restricting movement within society [23]. In the current pandemic, this has sometimes been described as a complete or enforced shutdown.

The concept of social distancing has sometimes been used interchangeably with physical distancing, which focuses on increasing the distance between individuals: for example, 1-2 meters or 6 feet. This helps to prevent droplet spread, which is assumed to be the main route of transmission [13, 20, 21]. One of the challenges with public health strategies focusing on enforced containment and restricting movement, is that they sometimes stand in contrast with human rights [22]. Strategies and measures based on community-wide shutdowns or isolation of individuals are also challenging, especially when taking into consideration the overall health and well-being of the individuals. It can result in individuals or families losing their livelihood, and, with this in mind, it is essential that these perspectives are considered and discussed to ensure a good adherence [22]. On top of this, the economic damage within society has been considered to decrease the overall health and increase the number of deaths by other causes, which is something that needs to be kept in mind when implementing infection control measures [24]. A more human centric approach could be to search for strategies that prevent the spread and focus on human behavior and needs, rather than the workings of the pathogen.

Emerging evidence shows that the main transmission takes place when individuals are interacting in close proximity, which is why increasing distance between individuals is of the utmost importance to slow transmission [20,21]. The ability to modify social behavior is, therefore, of great importance. Technology may offer some solutions within this context.

Within the current pandemic, our modern technological infrastructure has created new opportunities, which are unique in relation to historical pandemics. Technological infrastructures such as high-speed internet connection, digitalization of businesses and activities, and the broad adoption of technology within societies all over the globe, are able to aid the pandemic situation. For example, allowing 
people to socialize, work and study from home. This facilitates distancing between individuals but places less strain on those in isolation by enabling them to remain secluded but stay social. Technology can also support new ways of doing things and spark innovations which enable people to continue with certain activities - in particular activities that are important to overall health or to prevent poverty. For example, continuing to work or attend school, to have social contact, and access venues in a safer way. Access to technology has also contributed to new methods for collection of data and surveillance of contacts and disease spread. The adoption of smartphones has been a key factor in the current pandemic, enabling the development of a range of new approaches to tackle the spread. The use of smartphones during the Covid-19 pandemic is described briefly below.

\subsection{Mobile technologies to tackle Covid-19}

A number of mobile applications have been developed in many countries during this pandemic [25, 9]. The main targets of mobile technologies have been the following: symptom monitoring - by having users report in an app if they experience symptoms; quarantine control - using surveillance to ensure that users stay inside; flow monitoring/mobility reports by using aggregated data on human movement; and proximity based contact tracing - in order to identify and break chains of transmission [26].

The functionality and purpose of different solutions cover a varied spectrum from tracking infected people, issuing self-quarantine guidelines, performing surveys and providing citizens with the latest information [26]. Some apps collect not only the users' identity but also their location [9].

Many methods centered around contact tracing rely on Bluetooth to measure the distance between users, which is based on signal strength. This is a digitalized version of contact tracing - a classic but laborious epidemiological method [7]. Some of these approaches use a central software architecture, which includes central databases to identify users and to inform others about confirmed cases. The rapid development of these solutions have created much debate about privacy and ethics [25, 8, 7, 9]. Even those applications based on voluntary download have been criticized for not being fully in line with human rights [27].

Some applications which use contact tracing as an approach require that the user is tested by a healthcare professional. A positive test notifies others who have been in close proximity with the infected person within a specific time frame [26, 9]. However, even when data is anonymous, alerting individuals that they have been in contact with an infected person during a certain time period may enable them to identify that specific person. Identifying individuals, or groups of individuals, with a positive infection can be a sensitive issue and may cause stigma or even violence [28].

\subsection{Summary of challenges and opportunities within the situation}

A pandemic is a complex situation consisting of many interrelated elements, entities and relationships - as described in section 2. In summary, an intricate pandemic situation can be illustrated using the Rich Picture technique [29], as shown in Figure 1. The figure portrays the various levels of interaction and relationship between the elements and different entities.

In the upper middle part and the upper right side of the figure three of the main elements of a pandemic are illustrated: the infectious agent, the hosts and the transmission. The top left side illustrates the race to develop medical and pharmaceutical approaches to tackle the infectious agent. While the middle and bottom part of the figure illustrates the strategies and measures to reduce transmission in society, whilst balancing the effects on the economy and healthcare (middle of the figure), as well as the opportunities brought by digital and mobile technologies in terms of contact tracing and information sharing (right side of the figure).

Based on the understanding of the situation, we highlight that any approach to tackle a pandemic must be underpinned by evidence on how to minimize transmission. As the virus is primarily transferred via close contact between individuals, we have identified physical distance as one of the most important preventive measures to reduce transmission. This is also a key point in public health recommendations all over the globe [13, 30]. We conclude by re-defining this complex situation, from a problem focused on how to track the pathogen to a problem focused on awareness and distancing during social interactions. A design centered around physical distancing measures can increase focus on how to support human beings, especially if technology is used in more innovative ways. With this starting point, our proposed approach is slightly different from focusing on designs constructed to track infections and isolate individuals. We propose approaching the problematic situation by focusing on human behavior instead of the behavior of the pathogen. In the next section a design concept is proposed by first describing the theoretical background of influencing human behavior via 'nudging', followed by a description of the design concept. 


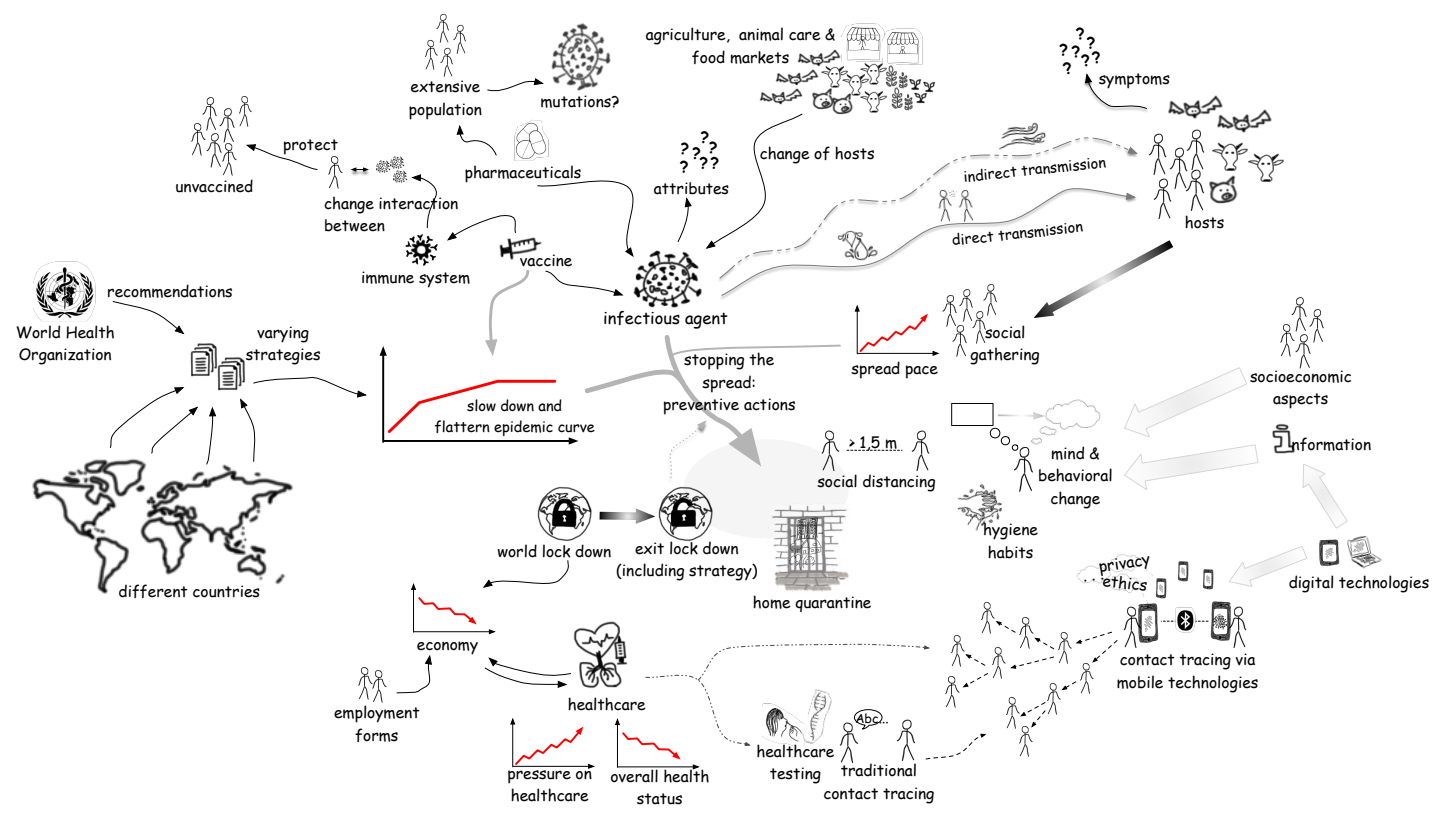

Figure 1. Current situation.

\section{Focusing on behavior in a pandemic}

During the recent decades the importance of human behavior has been increasingly recognized in the context of epidemics [31, 32]. This has led policy efforts to focus more on motivating healthy behavior and social distancing to aid in the reduction of disease risk. As people value the ability to stay socially active, a certain amount of risk is often accepted to gain social benefits. These risk trade-offs and social needs must be accounted for in the development of epidemic models and social distancing policies in order to efficiently understand and manage epidemics [32].

This has been even more evident during the Covid-19-pandemic, where social and behavioral science has been stated as a key factor to tackle the situation [33, 34, 35]. Due to the lack of vaccine, the importance of behavioral patterns such as hygiene routines, minimize crowding and social/physical distancing have been continuously promoted by authorities around the world.

Social distancing is challenging due to our nature as social beings [32]. Technology has provided some aid in the current pandemic by allowing us to communicate and, in many cases, work while staying home. However, most people would like to be able to meet others in real life, to leave their home, to attend events or get access to different environments, to go to work or school, and to meet friends, co-workers or family members. In the current situation, however, these actions carry the risk of transmitting or becoming infected by the virus. As transmission is possible from individuals without any or with very mild symptoms [19], it is of utmost importance that those who do interact in real life keep enough physical distance from others at all times [21].

To address this issue we need to re-design many of our social spaces. Many examples can be seen all over the world, such as signs in food stores or lines on the floor in different facilities. But we also need to re-design some of our fundamental behavior. In this case, mobile technology may offer some help. As most of us carry impressive computational devices on our person at all times, we can use these to continuously measure the distance between ourselves and others. This may relieve some of the cognitive load and the continuous attention required to maintain a safe distance, and thus enable us to take part in more social activities. A device with these capabilities can continuously assess our proximity to others, provide warnings when we get too close, assess our overall risk profile, and consequently also support and motivate us in changing our awareness and long-term habits. One approach within behavioral design is to use "nudges". These are small design features which can support individuals for example to pay attention to distance. Nudging is built on the idea that human beings have limited cognitive abilities, which at times prevent us from behaving in our best interest [36]. It is therefore proposed as a useful theoretical orientation for guiding the design of health behavior change support systems in the current 
pandemic situation.

\subsection{Designing behavior through nudging}

Nudging is an approach to improve behavior by making small changes in the context where choices are made. The concept was popularized by Thaler and Sunstein [36] in the area of behavioral economics but has since been used in a range of areas such as policy making and health interventions [36]. A nudge is a design element implemented in a particular context with the purpose to impact and improve choices made by individuals or groups. This approach is based on both philosophical ideas and research in behavioral economics and cognitive science [36].

The nudging approach brings about a significant paradigm shift in the understanding of human behavior. The shift moves from the neoclassical assumption that human beings are rational economic agents in decision making and that behavior can be observed as an expression of deliberate thought and individual preference, towards ideas that decision making is a product of two interacting systems of thought. These ideas can be categorized under the umbrella term Dual Process Cognitive Theories (DPTs). This theoretical orientation states that we have two types or systems of thinking: system 1 - the fast, intuitive and automatic system, and system 2 - the deliberate and slow system [37]. In decision making we tend to rely on the first system, which doesn't require as much cognitive effort, i.e. we go with the flow and act on cognitive heuristics. Furthermore, our thinking is often biased due to irrelevant factors provided by, for example, contextual, social and behavioral patterns. This causes our decision making to deviate from balanced, rational judgement - even during episodes of more deliberate thought. This deviation from rationality is often systematic, which makes us predictable and provides opportunity for manipulation regarding choices. The DPT paradigm has received more attention over the last decade due to Kahneman's [37] work. Thaler and Sunstein's [36] concept of nudging is built upon and expands this knowledge, and they also stipulate the ethical framework for how to use these insights in practice.

The primary ideas behind nudging includes that design is never neutral and the way we present things impact how people behave in that particular context. With this in mind, nudging is motivated to present things in a way which help improve decision making and promote good behavior, rather than leaving it to random. Furthermore, humans are not rational in regard to behavior, judgement and decision making, but instead are heavily influenced by contextual factors and biased in systematic ways $[36,38]$. To simplify, our behavior is continuously affected by the context, our cognitive limitations, and the fact that many choices we make are habitual and automatic. With the knowledge provided by behavioral science, nudges can be designed to make use of common cognitive bias and shortcuts and, as a result, make it easier for individuals to form better choices. In this way, nudges can be ethically motivated to complement the intention-action gap with the best interests of the individual in mind. Another important ethical guideline is that a nudge should be a small push to aid the 'right' decision. It should not, however, include coercion, reducing choice options or changing economic incentives in any significant way [36].

Hansen [38] has further clarified the concept of nudging by expanding on Thalers and Sunstein's [36] explanation. He describes a nudge as any attempt to influence people's behavior or judgement in predictable ways when cognitive boundaries, bias or habitual routines are posing barriers for them to make rational decisions or act in their own best interest. A nudge works by making use of these cognitive limitations to improve the situation [38]. This explanation provides more clarity to the definition of what a nudge is and what it is not, as it highlights the requirement of approaching cognitive limitations. For example, providing someone with information with the purpose of increasing knowledge is not a nudge. However, providing information as a reminder, or in order to increase attention, is a nudge because it focuses on cognitive limitations such as memory or attention [38].

\subsection{Nudge as a design element in a HBCSS context}

A system delivering nudges can be seen as an example of a behavior change support system. Behavior change support systems (BCSS) are persuasive systems [39] defined as: information systems designed to alter or form behavior or attitudes without deception or coercion [40]. In the context of health behavior change, these are referred to as health BCSS (HBCSS) [40]. In this paper the term nudge is considered a persuasive software feature, which can be implemented in a HBCSS. Both of these theoretical concepts are underpinned by the fundamental idea that design is never neutral. They are also compatible in terms of focus (e.g behavior change), as well as in ethical orientation by emphasizing transparency, voluntary choice and avoiding coercion $[40,36]$. Nudge-theory can contribute within the HBCSS context when design efforts tap into cognitive bias or limitations. For the design concept presented in 
this paper, we apply the concept of technology mediated nudging. The concept of technology mediated nudging is proposed for nudging strategies, when nudges are provided in a digital context to improve activities in the physical domain. To summarize, within this proposed strategy HBCSS is considered the context and type of system, technology mediated nudging is the strategy, and a nudge is the persuasive design element. In the following section we present a design concept for a health behavior change support system built on technology mediated nudging.

\subsection{Design concept}

We propose a design concept built on a framework of existing Bluetooth technology, for proximity measurements, but designed with a focus on individual behavior. The primary function of this approach would be to nudge the user to support and promote physical distancing, and the aim being to protect the user from disease by minimizing the risk of exposure to the virus. In contrast to many existing Covid-19 proximity applications, the software in this solution would be designed to notify individuals before they contract the virus and not after they might have already been exposed. Thereupon making this proposal a primary prevention approach. This concept can be applied to a range of Bluetooth devices, smartphones and smartwatches - to mention a few examples. In this paper, we focus on smartphones and suggest an application built around the notion of nudge theory.

\section{System requirements and context}

The first requirement for this system is that it is designed in a way that maintains respect for human rights and acts to support rather than track its user. The intention of this system is to avoid unnecessary tracking and centralized storage of health related data. All data will be encrypted and securely stored on the user's phone. The second requirement is that the intended outcome of this system should be underpinned by medical evidence and public health recommendations. In the current pandemic, physical distancing of 1 meter or more is one of the most important and evidence-based measures to significantly reduce the risk of viral transmission $[21,20]$.

Further requirements include certain technical and interactive properties. To be useful within a pandemic, the system needs to be readily available, easily accessible and scalable. This can be achieved by building on already existing hardware solutions. The suggested concept is also inspired by Apple and Google's Exposure notification API. The approach of this API is a decentralized software architecture, and all data is stored on users' phones. The possibility to offer the functionality to two of the biggest mobile platforms is a unique possibility and advantage. The suggested application will run in the background on the user's phone or other everyday wearable device. The app searches for other devices that have Bluetooth active. When contact with another individual occurs, the app takes note of the date, time and proximity by measuring the Bluetooth signal strength. If a user spends e.g. 1 minute or more in the vicinity of another device, and if the distance from the other device is for example $1.5 \mathrm{~m}$ or less, the user will get a notification (nudge). The default setting is that the user gets a notification every time in order to minimize the risk of disease transmission. However, an important design requirement is that the user can personalize the interaction with the system. The proposed approach is suitable in environments where people are free to move, and it needs to be considered in relation to all current an local public health strategies and recommendations. The concept is illustrated in Figure 2.

\section{Users and activities}

This system is intended for smartphone users who have the ability to download and adjust basic settings in the application. The app is designed to support the user during activities or in environments where people are at risk of getting too close to other individuals - for example at work, when spending time with friends, at restaurants or in schools. Moreover, the system is designed to support social interaction but also provide a framework to reduce the risk of disease transmission. As users may have different risk profiles or preferences, and different environments can increase or decrease the risk of transmission, this app will have settings to enable personalization. For example, warnings can be provided at different time intervals and can be based on a scale of proximity detection from 1 -- 3 meters. The requirement in order for the proximity detection to work is that all parties have their Bluetooth activated. This can be requested by users when planning social activities, or it can be promoted in certain environments by information signs.

\section{Nudging for behavior change}

The intention behind this application concept is to support users in adapting and maintaining safe behavior by providing nudges which target both systems (system 1 and 2) involved in decision making (see section 3.1). These nudges are described and categorized below in relation to the outcome-change matrix $(\mathrm{O} / \mathrm{C}$ matrix $)$ which is a key construct in the BCSS concept [40]. The $\mathrm{O} / \mathrm{C}$ matrix offers a 2-dimensional categorization 


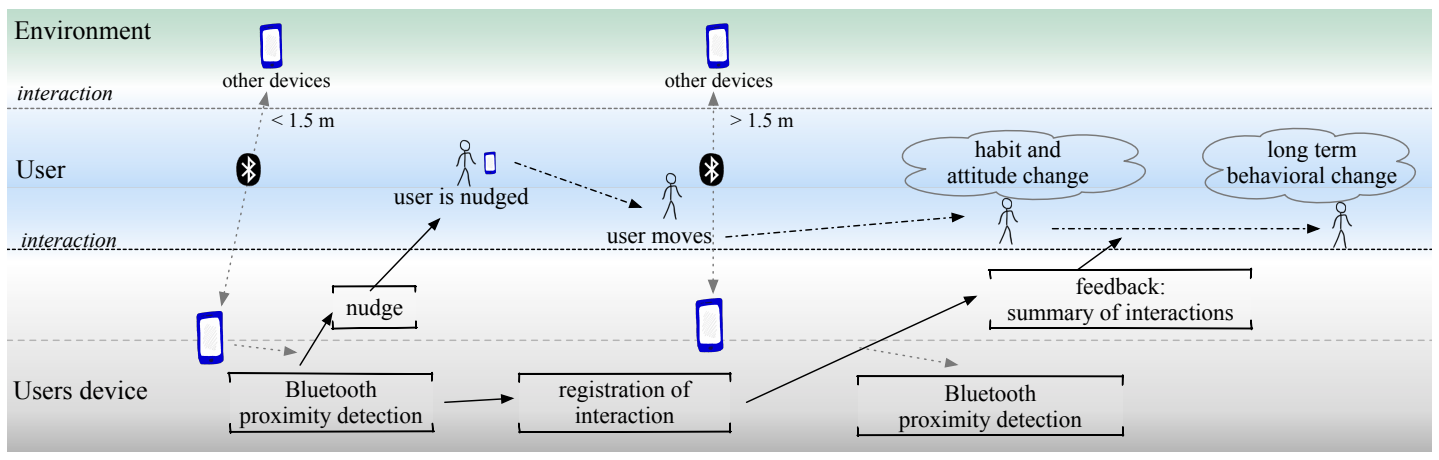

Figure 2. Design concept building on nudging.

based on the intended outcome (reinforcing, altering, or forming a behavior or attitude) and type of change (e.g behavior, compliance or attitude) [40].

The primary and most important feature of this system is to provide a notification - a short term nudge -- if the user becomes too close to another individual. This notification is designed to result in a direct and automatic impact on behavior - therefore it is named a system 1 nudge. The intention is to remind the user to take note of holding distance and thus reducing the risk of exposure, or exposing others, to the virus. As such, this is a primary prevention approach. The behavioral change targeted in this context is defined as a B-change in the $\mathrm{O} / \mathrm{C}$ matrix. Different users may require altering a behavior $(\mathrm{A} / \mathrm{B})$ re-enforcing it $(\mathrm{R} / \mathrm{B})$ or forming a new behavior $(\mathrm{F} / \mathrm{B})$, depending on their previous habits. This highlights the need for personalized settings. The primary route in order to enable the necessary change in behavior is to deliver short direct persuasive messages - system 1 nudges - aimed at tapping into the user's automatic and intuitive system of decision making. In this case, the system 1 nudges approach the user's cognitive limitations such as attention and memory, and with nudge repetition, habitual and long term behavioral change may occur. Nudges can be set to notify the user, with both sound and vibration, after a predefined time frame. The distance and time limits can be set by the user, and can be based on their risk profile. Users will be able to exclude certain devices found in their household and those used by other family members. It is important that the solution offers ways to mute, stop tracking or include time slots where tracking is irrelevant, for example when individuals work closely with others but wear protective equipment.

Secondly, the application offers the user long term support via nudges targeting the slow and deliberate system of decision making. System 2 nudges aim to promote and reinforce positive attitude towards physical distancing. This is categorized as R/A (reinforcing attitudes) in the $\mathrm{O} / \mathrm{C}$ matrix and can further facilitate long term behavioral change. Reinforcing attitudes may impact behavior such as taking part in social activities. The design can offer, for example, a summary of the number of close proximity interactions on a daily or weekly basis. It can support the user in planning by providing a risk profile or motivational feedback, as well as providing tips to minimize the number of close interactions. It can also provide elements of gamification or enable the user to compare progress with others (social nudging). How and when this information is presented to the user and how to design the interactive details, needs to be further developed in user-orientated research.

\section{Discussion and future work}

Managing a pandemic, when a new pathogen is involved, is not an easy or straightforward process. The human element adds to the complexity as strategies need to offer balance between slowing down transmission and avoiding harm to the society. Innovative strategies are needed to complement existing ones, not only because pandemics will continue to occur and are on the increase, but also because society is changing.

We propose a different approach for how mobile solutions can be used in epidemic situations. An approach built on existing technology centered around proximity estimation, but in our case used as a primary prevention tool to support physical distancing by focusing on human behavior. Furthermore, it is based on the current understanding of main transmission routes and the efficiency physical distancing has as a preventative measure [21]. Consequently, this approach can be applied for various types of technology and designs and can be relevant within epidemic contexts involving pathogens with similar transmission modes. We propose that more research involving mobile technology for pandemic situations continues to pursue 
the direction of research where human behavior is in the focus of attention. After all, targeting human behavior is paramount when managing infectious disease outbreaks $[31,32,33]$.

With this proposal, we have developed a conceptual design model based on nudging to support and promote physical distancing. By adopting this approach, we can assume a lower risk of exposure for the user as well as individuals in the vicinity of the user. Consequently, this approach has the potential to lower transmission rates within a population where a number of individuals use the system. This design focuses on the same goals as, for example, contact tracing apps, but does not require testing capacity, identification of individuals with symptoms or involvement from the health care. This is a strength with this approach. A weakness, shared with contract tracing applications, is that it relies on the Bluetooth proximity estimation to be accurate.

Our concept stems from design thinking and exercises a fresh perspective - from the pathogenic focus of disease tracking towards a spotlight focused more on human behavior and our needs for social interaction. It is with this shift in mindset that we can fully utilize technology within a population living through an epidemic.

An important point to consider, and one that poses a possible risk, is that the use of technology to minimize exposure may cause individuals to become too relaxed. PPIs (personal protective equipment) has been the subject of similar discussions. This needs to be addressed and the responsibility of the individual needs to be clearly stated with user information. It is also important to state that there is no such thing as a completely safe distance, especially in environments with risk of aerosol transmission. However, it is possible to mitigate some of these risks by providing information and recommendations about behavior in different environments, as well as opportunities to provide personalized settings. On the other hand, the proposed system may also support the formation of new habits, and enable individuals to keep distance even when they are not using the system.

Further research is planned to evaluate ideas and continue development of the design. To involve potential users is crucial to refine and evaluate the concept. Furthermore, there are decisions that need to be considered in the development of this system, and how to balance privacy and accuracy issues. For example, the application's capability of logging interaction with other devices. Storing identification will improve the accuracy of statistics administered to the user, and also minimize nudges from repeated interactions. This could be solved if the other users have installed the same application, but this will lead to challenges with enough up-take. This solution is also somewhat more sensitive from a privacy standpoint. Another direction would be to ensure there is no logging, but that the focus is solely on achieving health behavior change. This may lead to repeated nudges for the same person, which will result in daily/weekly summary misrepresentation less a presentation of the unique individual but instead the number of close person-to-person interactions. The benefit is that this solution is less privacy invasive and does not require that the same app is used by others. It only requires that all parties have an active Bluetooth. Furthermore, repeated nudges may be beneficial from a habitual standpoint. Users will be involved in taking these decisions.

\section{References}

[1] Y.-R. Guo, Q.-D. Cao, Z.-S. Hong, Y.-Y. Tan, S.-D. Chen, H.-J. Jin, K.-S. Tan, D.-Y. Wang, and Y. Yan, "The origin, transmission and clinical therapies on coronavirus disease 2019 (covid-19) outbreak-an update on the status," Military Medical Research, vol. 7, no. 1, pp. 1-10, 2020.

[2] World Health Organisation, "Timeline of who's response to covid-19," 2020. https: //www.who.int/news-room/detail/ 29-06-2020-covidtimeline, Last accessed on 2020-07-13.

[3] Eurosurveillance Editorial Team and others, "Note from the editors: World health organization declares novel coronavirus (2019-ncov) sixth public health emergency of international concern," Eurosurveillance, vol. 25, no. 5, p. 200131e, 2020.

[4] J. Pierre, "Nudges against pandemics: Sweden's covid-19 containment strategy in perspective," Policy and Society, pp. 1-16, 2020.

[5] Eurosurveillance editorial team and others, "Rapid risk assessment from ecdc: Resurgence of reported cases of covid-19 in the eu/eea, the uk and eu candidate and potential candidate countries," Eurosurveillance, vol. 25, no. 26, p. 2007021, 2020.

[6] J. L. Guest, C. del Rio, and T. Sanchez, "The three steps needed to end the covid-19 pandemic: bold public health leadership, rapid innovations, and courageous political will," JMIR Public health and surveillance, vol. 6, no. 2, p. e19043, 2020.

[7] M. J. Parker, C. Fraser, L. Abeler-Dörner, and D. Bonsall, "Ethics of instantaneous contact tracing using mobile phone apps in the control of the covid-19 pandemic," Journal of Medical Ethics, 2020.

[8] M. Ienca and E. Vayena, "On the responsible use of digital data to tackle the covid-19 pandemic," Nature medicine, vol. 26, no. 4, pp. 463-464, 2020.

[9] R. A. Fahey and A. Hino, "Covid-19, digital privacy, and the social limits on data-focused public health responses," International Journal of Information Management, vol. 55, p. 102181, 2020.

[10] R. M. Elavarasan and R. Pugazhendhi, "Restructured society and environment: A review on potential technological strategies to control the covid-19 
pandemic," Science of The Total Environment, p. 138858, 2020.

[11] T. Brown et al., "Design thinking," Harvard business review, vol. 86, no. 6, p. 84, 2008.

[12] C. Dosi, F. Rosati, M. Vignoli, et al., "Measuring design thinking mindset," in DS 92: Proceedings of the DESIGN 2018 15th International Design Conference, pp. 1991-2002, 2018.

[13] CDC, National Center for Immunization and Respiratory Diseases (NCIRD), Division of Viral Diseases, "Social distancing: Keep a safe distance to slow the spread," 2020. https://www.cdc.gov/coronavirus/ 2019-ncov/prevent-getting-sick/ social-distancing.html, Last accessed 2020-07-13.

[14] M. Reynolds and S. Holwell, Systems approaches to managing change: a practical guide. Springer, 2010.

[15] R. Bonita, R. Beaglehole, and T. Kjellström, Basic epidemiology. World Health Organization, 2006.

[16] C. S. Brown and N. William, "Communicable diseases," in Global health (B. D. Nicholson, J. McKimm, and A. K. Allen, eds.), pp. 124-141, Los Angeles, Calif. SAGE, 2016.

[17] Z.-W. Ye, S. Yuan, K.-S. Yuen, S.-Y. Fung, C.-P. Chan, and D.-Y. Jin, "Zoonotic origins of human coronaviruses," International journal of biological sciences, vol. 16, no. 10, p. 1686, 2020.

[18] Z. Wu and J. M. McGoogan, "Characteristics of and important lessons from the coronavirus disease 2019 (covid-19) outbreak in china: summary of a report of 72314 cases from the chinese center for disease control and prevention," Jama, vol. 323, no. 13, pp. 1239-1242, 2020 .

[19] S. M. Moghadas, M. C. Fitzpatrick, P. Sah, A. Pandey, A. Shoukat, B. H. Singer, and A. P. Galvani, "The implications of silent transmission for the control of covid-19 outbreaks," Proceedings of the National Academy of Sciences, 2020.

[20] C. R. MacIntyre and Q. Wang, "Physical distancing, face masks, and eye protection for prevention of covid-19," The Lancet, 2020.

[21] D. K. Chu, E. A. Akl, S. Duda, K. Solo, S. Yaacoub, H. J. Schünemann, A. El-harakeh, A. Bognanni, T. Lotfi, M. Loeb, et al., "Physical distancing, face masks, and eye protection to prevent person-to-person transmission of sars-cov-2 and covid-19: a systematic review and meta-analysis," The Lancet, 2020.

[22] J. A. Singh, "Global health governance and ethics," in An introduction to global health ethics (A. D. Pinto and R. E. G. Upshur, eds.), pp. 58-72, New York, USA: Routledge, 2013.

[23] A. Wilder-Smith and D. O. Freedman, "Isolation, quarantine, social distancing and community containment: pivotal role for old-style public health measures in the novel coronavirus (2019-ncov) outbreak," Journal of travel medicine, vol. 27, no. 2, p. taaa020, 2020.

[24] S. Balkin and J. F. McDonald, "Towards a model of optimal social distancing during a pandemic," Available at SSRN 3616229, 2020.

[25] J. Morley, J. Cowls, M. Taddeo, and L. Floridi, "Ethical guidelines for covid-19 tracing apps," 2020.
[26] U. Gasser, M. Ienca, J. Scheibner, J. Sleigh, and E. Vayena, "Digital tools against covid-19: taxonomy, ethical challenges, and navigation aid," The Lancet Digital Health, 2020.

[27] Amnesty International, "Bahrain, Kuwait and Norway contact tracing apps among most dangerous for privacy," 2020. https://www . amnesty.org/en/latest/news/2020/06/ bahrain-kuwait-norway-contact-tracing -apps-danger-for-privacy, Last accessed 2020-07-14.

[28] C.-Y. Lin et al., "Social reaction toward the 2019 novel coronavirus (covid-19)," Social Health and Behavior, vol. 3, no. 1, p. 1, 2020.

[29] P. Checkland and J. Poulter, "Soft systems methodology," in Systems Approaches to Making Change: A Practical Guide, pp. 201-253, Springer, 2020.

[30] World Health Organisation, "Covid-19: physical distancing," 2020. https://www.who.int/ westernpacific/emergencies/covid-19/ information/physical-distancing, Last accessed on 2020-07-14.

[31] S. Funk, M. Salathé, and V. A. Jansen, "Modelling the influence of human behaviour on the spread of infectious diseases: a review," Journal of the Royal Society Interface, vol. 7, no. 50, pp. 1247-1256, 2010.

[32] E. P. Fenichel, C. Castillo-Chavez, M. G. Ceddia, G. Chowell, P. A. G. Parra, G. J. Hickling, G. Holloway, R. Horan, B. Morin, C. Perrings, et al., "Adaptive human behavior in epidemiological models," Proceedings of the National Academy of Sciences, vol. 108, no. 15, pp. 6306-6311, 2011.

[33] P. D. Lunn, C. A. Belton, C. Lavin, F. P. McGowan, S. Timmons, and D. A. Robertson, "Using behavioral science to help fight the coronavirus," Journal of Behavioral Public Administration, vol. 3, no. 1, 2020.

[34] P. D. Lunn, S. Timmons, H. Julienne, C. Belton, M. Barjaková, C. Lavin, and F. McGowan, "Using decision aids to support self-isolation during the covid-19 pandemic," ESRI WORKING PAPER No. 664, 2020.

[35] G. Lawton, "Best behaviour," New Scientist, vol. 246, no. 3281, pp. 38-42, 2020.

[36] R. H. Thaler and C. R. Sunstein, Nudge: Improving decisions about health, wealth, and happiness. Penguin, 2009.

[37] D. Kahneman, Thinking, fast and slow. Macmillan, 2011.

[38] P. G. Hansen, "The definition of nudge and libertarian paternalism: Does the hand fit the glove?" European Journal of Risk Regulation, vol. 7, no. 1, pp. 155-174, 2016.

[39] B. J. Fogg, "Persuasive technology: using computers to change what we think and do," Ubiquity, vol. 2002, no. December, p. 2, 2002.

[40] H. Oinas-Kukkonen, "A foundation for the study of behavior change support systems," Personal and ubiquitous computing, vol. 17, no. 6, pp. 1223-1235, 2013. 